ScienceDirect

www.sciencedirect.com

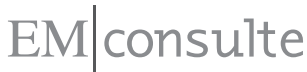

www.em-consulte.com

Neuropsychiatrie de l'enfance et de l'adolescence 64 (2016) 498-507

neuropsychiatrie

de l'enfance

et de l'adolescence

Cas clinique

\title{
Quand l'enfant révèle un abus sexuel : le vécu du couple, ses fonctions et conséquences
}

\section{When the child reveals a sexual abuse: The real-life experience of the couple, its functions and the consequences}

\author{
A. Gallo*, C. Wertz, A. Blavier \\ Université de Liège, Liège, Belgique
}

\begin{abstract}
Résumé
Cette étude a pour objectif principal d'explorer les difficultés rencontrées par le couple conjugal et parental lorsqu'il apprend que l'un de ses enfants a été abusé sexuellement. Cet abus peut être intra- ou extrafamilial. Le vécu du couple est un domaine qui mérite d'être davantage investigué car nous savons combien la souffrance des parents influence l'état de santé mentale de l'enfant et peut alimenter voire maintenir les symptômes de ce dernier. Notre échantillon comprend six couples dont l'enfant a été victime d'un abus sexuel. Ceux-ci ont été recrutés au sein des équipes SOS-Enfants. Un design expérimental de type qualitatif a été mis en place : nous avons rencontré les couples à une ou deux reprises. Lors de ces rencontres, nous avons réalisé un entretien semi-structuré afin de récolter des informations démographiques sur les caractéristiques de l'abus. Le Life Events and Difficulties Schedule (Brown et Harris, 1978), a permis d'inventorier les évènements potentiellement traumatiques survenus lors des six derniers mois. Nous avons également administré cinq questionnaires et dirigé un entretien semi-structuré construit dans le cadre de cette recherche sur base de la littérature scientifique. Ceux-ci comprennent l'Échelle d'Ajustement Dyadique (Spanier, 1976), l'Inventaire de Coping Dyadique (Bodenmann, 2008), l'Inventaire d'Alliance Parentale (Lacharité, 1996), le Questionnaire d'Auto-Évaluation de la Compétence Parentale (Terrisse et Trudelle, 1988) et enfin l'Inventaire des Désirs Sexuels (Spector et al., 1996). Ces questionnaires visent chacun à évaluer une dimension plus spécifique du couple. Les résultats obtenus soulignent une détresse chez chaque couple. Cependant, celle-ci s'exprime de façon différente, aussi bien entre les couples qu'entre les partenaires conjugaux eux-mêmes. Néanmoins, pour chacun d'eux, la dimension de la parentalité semble être une ressource leur permettant de s'investir auprès de leur enfant victime. Cet investissement prend la forme d'une focalisation sur l'enfant ayant pour conséquence l'effacement du couple conjugal. En effet, le temps passé ensemble, les moments en couple et l'intimité diminuent. Si certains couples manifestent néanmoins un rapprochement, celui-ci se fait autour de l'enfant et du problème. Cette capacité à faire équipe nous laisse penser que ces couples disposent de ressources pour faire face à l'abus. L'intérêt porté à la détresse conjugale et parentale semble donc primordial car cette étude montre que les parents, s'ils tentent souvent dans un premier temps de mettre en place des mécanismes assurant l'homéostasie et la sécurité familiales, ceux-ci ne sont pas toujours adaptatifs à long terme. Nous voyons alors un risque de créer un secret de famille, lourd à porter, ou encore une surprotection figeant l'enfant dans son rôle de victime, pouvant en outre provoquer un étiolement de la dynamique conjugale. Dès lors, il nous semble impératif de pouvoir également soutenir la conjugalité par des interventions cliniques. Cependant, d'autres études portant sur un plus grand échantillon sont à ce jour encore nécessaires afin de mettre en évidence, à une plus large échelle, les difficultés vécues par ces couples. (C) 2016 Elsevier Masson SAS. Tous droits réservés.
\end{abstract}

Mots clés : Abus sexuel ; Conjugalité ; Parentalité ; Détresse parentale ; Modélisation

\section{Abstract}

This study has for objective to explore the difficulties faced by the conjugal couple and parental when he learns that one of his children had been sexually abused. This abuse can be perpetrated inside or outside the family. The experience of the couple is an area that deserves to be further investigated because we know how the suffering of parents influence the state of mental health of the child and can power or to keep symptoms of the latter. Our sample includes six couples whose child has been victim of sexual abuse. They have been recruited within the SOS-Enfants

\footnotetext{
* Auteur correspondant.

Adressese-mail:alicia.gallo@ulg.ac.be (A.Gallo), celine.wertz@ulg.ac.be (C. Wertz), adelaide.blavier@ulg.ac.be (A. Blavier).
} 
teams. An experimental design of qualitative type has been implemented: we met couples on one or two occasions. In these meetings, we realized a semi-structured interview to gather demographic information about the characteristics of the abuse. The Life Events and Difficulties Schedule (Brown and Harris, 1978) has allowed to identify potentially traumatic events that occurred during the last six months. We also administered five questionnaires and conducted a semi-structured interview built as part of this research based on the scientific literature. These include the Scale of Adjustment Dyadic (Spanier, 1976), the Inventory of Dyadic Coping (Bodenmann, 2008), the Inventory of Parental Alliance (Lacharité, 1996), the Self-Assessment Questionnaire of the Parental Competence (Terrisse et Trudelle, 1988) and finally the Inventory of Sexual Desires (Spector et al., 1996). These questionnaires aim to assess a specific dimension of the couple. The results underline distress in each couple. However, this is expressed differently, both between couples that between the marital partners themselves. However, for each of them, the dimension of parenting seems to be a resource enabling them to invest with their child victim. This investment takes the form of a focus on the child resulting in the erasure of the conjugal couple. Indeed, the time spent together, the couple moments and intimacy decrease. If some couples nevertheless demonstrate a rapprochement, this is done around the child and the problem. This ability to make team suggests that these couples have resources to cope with the abuse. The interest on conjugal and parental distress seems essential as this study shows that parents, if they try often as a first step to implement the mechanisms for homeostasis and family security, they are not always adaptive in the long term. We then see a risk of creating a secret family, heavy to carry or overprotection freezing child in his role of victim, can also cause a weakening of the marital dynamics. Therefore, believe us it is imperative to also support the conjugality by clinical interventions. However, other studies involving a larger sample are to this day still needed to highlight the difficulties experienced by these couples on a wider scale.

(C) 2016 Elsevier Masson SAS. All rights reserved.

Keywords: Child sexual abuse; Conjugality; Parenthood; Parental distress; Modeling

\section{Introduction}

Selon une étude de Grosz et al. [1], le fonctionnement des enfants, et plus largement celui des familles, est gravement mis en péril lorsqu'il y a eu un abus sexuel. Manion et al. [2] nous expliquent que la révélation a le pouvoir de traumatiser une famille entière. L'équipe Kaléidos [3] affirme que la révélation est une crise dans l'homéostasie du système familial. Barudy [4] précise que les réactions des familles dépendent de leur niveau de fonctionnement avant l'évènement.

Il est important de préciser ce que nous entendons par «l'abus sexuel envers un enfant ». Selon Tourigny et Baril [5], de façon consensuelle, il semble compliqué de définir l'agression sexuelle envers les enfants. Reprenons la définition qui permettrait de réunir les différents points qu'il est possible de trouver dans la littérature scientifique ([5], p.9) : «l'agression sexuelle contre un enfant est définie comme tout acte ou jeu sexuel, hétérosexuel ou homosexuel, entre une ou des personnes en situation de pouvoir, d'autorité ou de contrôle, et un enfant mineur. Ces actes sexuels ont pour but de stimuler sexuellement l'enfant ou de l'utiliser pour se stimuler soi-même sexuellement ou pour stimuler une autre personne. Lorsqu'il s'agit d'un adulte ou d'une personne ayant de trois à cinq ans de plus que la victime, les lois de plusieurs pays prévoient qu'il y a automatiquement une situation de pouvoir et qu'il s'agit donc d'agression sexuelle. Lorsqu'il n'y a pas de situation de pouvoir ou de contrôle, il y a agression sexuelle si la victime ne consent pas à l'activité sexuelle. » On peut différencier les agressions sexuelles sur un enfant selon qu'elles soient intra- ou extrafamiliales. On parle d'agressions sexuelles intrafamiliales lorsque les protagonistes ont un lien de parenté, même large. Les agressions sexuelles extrafamiliales, quant à elles, concernent des personnes sans lien de parenté mais sans être nécessairement inconnues à la victime. Il est estimé, grâce à des études nord-américaines, qu'au moins une femme sur cinq, et un homme sur dix ont vécu une agression sexuelle durant l'enfance ou l'adolescence [6]. Cependant, un grand nombre d'enfants ne dévoilent pas les faits dont ils ont été victimes. Le dévoilement est défini par Martin et Van Poppel ([7], p.36) comme la « révélation verbale ou comportementale par l'enfant, d'un abus sexuel dont il est, a été ou croit avoir été victime ou dont le tiers qui observe et donc interprète l'expression de l'enfant croit ou suspecte celui-ci d'en avoir été victime. » Palmer et al. [8] identifient que dans $90 \%$ des cas, les enfants disent être contents d'avoir révélé les faits, car ils passent du secret à l'ouverture. Parfois, cette révélation met un terme à l'abus et résout un conflit interne et les victimes s'apaisent d'une certaine culpabilité. Dans ce processus de dévoilement, la réaction parentale a toute son importance. En effet, l'enfant obtenant du soutien de ses parents peut se sentir protéger des risques liés à l'agression subie grâce à la diminution de l'appréhension du danger en lien avec les faits [9]. Ainsi, nous comprenons pourquoi il est intéressant de se pencher sur l'impact de cette révélation sur le couple.

De nombreuses études portent sur l'impact du dévoilement d'un abus sexuel sur les parents d'enfants ayant été victimes. Deux recherches $[10,11]$ nous montrent que les parents vivent un grand nombre de difficultés à la suite de cette révélation. En effet, un stress psychologique est vécu par ces derniers. Celui-ci s'apparenterait à un traumatisme secondaire. Cependant, Kilroy et al. [12] précisent qu'il ne s'agit pas tout à fait d'un trauma vicariant ni d'un deuil. Il semblerait que l'impact émotionnel soit directement dû à l'annonce de l'abus ainsi qu'à la vie quotidienne, au contexte familial (difficultés relationnelles, diminution du support, augmentation de la détresse psychique, problèmes financiers et organisation teintée de pression), aux pensées intrusives des parents et aux changements dans la dynamique familiale (haut niveau d'expression des émotions, frontières diffuses, surprotection, symptômes de l'enfant, 
support du système professionnel, pertes relationnelles). Cyr et al. ([13], p.255) parlent d'une «blessure affective profonde » qui plonge les parents dans le doute et le déni, et qui s'apparente à un travail de deuil. En 2001, dans une méta-analyse, Elliot et Carnes [14] mettent en évidence des symptômes d'ESPT significativement élevés pour les parents, tant pour la mère que pour le père, et ce, pendant deux années. Cependant, une étude menée par Kelley en 1990 [15] met en évidence que les symptômes sont deux fois plus élevés chez les mères d'enfants victimes d'abus que chez les pères. Davies [10] différencie qualitativement les symptômes d'ESPT des mères et des pères : les mères font plus preuve de pensées intrusives et d'évitement alors que les pères, s'ils évitent tout autant, manifestent moins d'intrusions. De plus, Van Toledo et Seymour [16] décrivent des réactions émotionnelles importantes : colère, désespoir, déni, ambivalence, culpabilité,... Des informations empiriques [3] indiquent qu'il faut penser les parents comme des victimes secondaires. Ils éprouvent des complications dans leur rôle de parent face à l'enfant en difficulté avec une perte de repères éducatifs. Le lien entre les parents est mis à mal. D'ailleurs, Davies [10] rapporte que les scores de satisfaction conjugale indiquent qu'il est nécessaire d'avoir recours à des interventions. Kilroy et al. [12] ajoutent que les parents évitent les moments d'intimité car cela fait référence à l'abus, ils marquent une distance émotionnelle. Une étude de Myrick et Green [17] avance les mêmes conclusions en ajoutant que les parents d'enfants victimes sont aussi traumatisés, bien qu'ils minimisent les faits, tout comme les professionnels, pour aider l'enfant avant tout.

Cependant, ces études traitent des symptomatologies vécues par les parents selon une perspective individuelle, sans se pencher sur la dynamique relationnelle, tant conjugale que parentale. Or, au vu des difficultés individuelles, il est légitime de penser que cette dynamique est également affectée. De plus, ces études n'explicitent pas clairement le vécu de ces couples. Ainsi, il est compliqué de comprendre ce qu'expérimente réellement le couple conjugal et parental suite à la révélation d'un abus sexuel par l'un de leurs enfants. Ici réside l'intérêt de cette recherche. Nous savons en effet combien la souffrance parentale peut influencer voire maintenir la symptomatologie de l'enfant à la suite d'un évènement potentiellement traumatique [18], à l'inverse, une réaction parentale adaptée peut aider l'enfant.

L'objectif de cette recherche est donc d'explorer - à travers les récits - les difficultés, les ressources, les besoins que le couple conjugal et parental vit lorsqu'il apprend que l'un de ses enfants a été abusé sexuellement mais aussi d'inscrire ces difficultés dans une temporalité.

Nous nous attendons à ce qu'il apparaisse, au sein du couple, une détresse significative après l'annonce de l'abus sexuel vécu par un enfant. Nous avons subdivisé cette hypothèse générale en six sous-hypothèses, relatives à chacun des sous-systèmes. Au niveau du couple conjugal, nous nous attendons à observer une insatisfaction conjugale, des difficultés d'ajustement dyadique ainsi qu'une baisse de l'intimité. Au sein du couple parental, nous pensons observer une insatisfaction dans le rôle parental, un sentiment d'incompétence parentale et enfin, un affaiblissement de l'alliance parentale.

\section{Méthodologie}

\subsection{Participants}

Nous avons rencontré six couples dont un ou plusieurs enfants avaient été abusés sexuellement. Pour quatre d'entre eux, les abus avaient eu lieu dans l'année et pour les deux autres, les abus remontaient à trois ans. Cet abus était soit commis par un membre externe à la famille (couple 1) soit interne à celleci (pour l'ensemble des cinq autres couples), l'abuseur n'étant jamais un membre du couple rencontré. Ces couples ont été recrutés avec la collaboration des équipes SOS-Enfants, équipes spécialisées dans la prise en charge des situations de maltraitances d'enfants. Une (couples 2, 4 et 6 ) à deux (couples 1, 3 et 5) rencontres ont eu lieu. Seuls les membres du couple étaient vus.

Le couple 1 est composé d'un homme de 44 ans et d'une femme de 37 ans. Ils ont un enfant unique de 9 ans. L'abus vécu se caractérise par des attouchements de la part d'un mineur de 4 ans son aîné lors d'un week-end de scoutisme.

Le couple 2 est une famille recomposée. Les membres du couple sont âgés de 40 ans, ils ont en commun une fille de 8 ans. La victime, fille d'une première union de la mère est âgée de 16 ans. Les abus ont été perpétrés par un grand oncle paternel durant plusieurs années.

Le couple 3 a deux enfants : une fille de 6 ans et un garçon de 15 mois. Les parents sont âgés de 35 ans. La fillette a été victime d'attouchements de la part d'un cousin maternel éloigné. Les faits ne se seraient produit qu'une seule fois.

Le couple 4 est constitué d'un homme de 39 ans et d'une femme de 33 ans. Ils ont deux enfants : un aîné de 13 ans et une cadette de 7 ans. Des jeux sexuels se sont produits entre le frère et la sœur durant plusieurs mois.

Le couple 5 est recomposé. Madame est âgée de 37 ans et Monsieur de 28 ans. Madame a de son côté 3 enfants et Monsieur a un enfant. Les abus se seraient produits entre deux enfants de Madame.

Le couple 6 est composé d'un homme âgé de 35 ans et d'une femme âgée de 31 ans. Leurs deux fils ayant moins de 10 ans ont été victimes d'attouchements de la part d'un cousin paternel. Les abus ont duré plusieurs mois.

\subsection{Procédure}

Durant l'entretien, nous récoltions une série d'informations démographiques concernant les circonstances de l'abus mais aussi les données familiales. Ensuite, nous parcourions le Life Events and Difficulties Schedule (Brown et Harris, 1978) qui nous permettait d'évaluer si d'autres évènements potentiellement traumatiques étaient survenus durant les six derniers mois afin d'augmenter la validité discriminante de l'étude. Ensuite, les participants complétaient individuellement cinq questionnaires évaluant chacun une dimension particulière du couple. Nous avons utilisé l'Échelle d'Ajustement Dyadique (Spanier, 1976) ; l'Inventaire d'Ajustement Dyadique (Bodenmann, 2008) ; l'Inventaire d'Alliance Parentale (Lacharité, 1996) ; 
le Questionnaire d'Auto-Évaluation de la Compétence Éducative et Parentale [19] et enfin, l'Inventaire du Désir Sexuel (Spector, Carey, et Steinberg, 1996). Pour terminer l'entretien, nous parcourions une grille créée spécifiquement pour cette recherche grâce à la revue de la littérature, permettant de parcourir l'ensemble des domaines susceptibles d'être affectés par la révélation de l'abus sexuel vécu par un enfant.

\subsection{Mesures}

\subsubsection{Le Life Events and Difficulties Schedule (LEDS)}

Cette échelle, créée par Brown et Harris (1978), se présente sous la forme d'un entretien semi-structuré qui passe en revue un ensemble de facteurs de stress qui peuvent être survenus dans les six derniers mois. Les participants sont alors amenés à dire si ces évènements leur sont arrivés. Dans la version originale, les participants doivent raconter l'évènement en détail. Ici, nous nous sommes limités à savoir si l'évènement avait eu lieu.

\subsubsection{L'Échelle d'Ajustement Dyadique (DAS)}

L'échelle, créée par Spanier (1976), évalue quatre dimensions théoriques de l'ajustement dyadique : le consensus dyadique, la satisfaction, la cohésion et l'expression d'affection. Ces quatre dimensions relèvent de la sphère conjugale. Cet outil a été utile pour révéler une éventuelle détresse du couple conjugal. Il existe un score seuil de 97 en-dessous duquel la relation est dite « en détresse » (Jacobson et al., 1987). La version française a été validée par Baillargeon et al. en 1986 [20].

\subsubsection{L'Inventaire d'Ajustement Dyadique (DCI)}

La version française (Bodenmann, 2008) de cet inventaire a été validée. Cette échelle évalue comment le couple gère les évènements stressants à travers six dimensions : la communication à propos du stress, l'ajustement dyadique positif ou soutenant, l'ajustement dyadique négatif, l'ajustement dyadique délégué, l'ajustement conjoint et enfin, l'évaluation que le répondant fait de l'ajustement dyadique de son couple. Cet outil va donc fournir des informations concernant les éventuelles difficultés pour les membres du couple à gérer le stress de la révélation ensemble. Bodenmann (2000) [21] répartit les scores en trois catégories. Premièrement, la catégorie moyenne se situant de 111 à 145 , ce qui correspond à un coping dyadique adéquat. Deuxièmement, la catégorie inférieure signifie que le couple éprouve des difficultés à gérer une situation de stress ensemble (en-dessous de 111), et enfin la catégorie supérieure (au-dessus de 145).

\subsubsection{L'Inventaire d'Alliance Parentale (PAI)}

Ce questionnaire a été créé par Abidin (1988) et traduit en français par Lacharité (1996). Il évalue le degré d'engagement et de coopération des membres du couple en ce qui concerne les comportements de parentage. Ces items permettent d'évaluer jusqu' à quel degré, l'un des parents croit avoir une bonne relation avec l'autre parent quant aux responsabilités visant l'éducation et les soins de l'enfant. Cet outil éclaire quant à la façon dont les parents se soutiennent l'un l'autre dans leurs interactions avec l'enfant. Le score varie de 20 à 100 . Plus il est élevé et plus le parent a une perception favorable de l'équipe qu'il forme avec son partenaire pour répondre aux différentes fonctions parentales.

\subsubsection{Le Questionnaire d'Auto-Évaluation de la Compétence Éducative et Parentale (QAECEP)}

Cet outil est l'adaptation française par Terrisse et Trudelle [19] du « Parenting Sense of Competence Scale » élaboré par Gibaud-Wallston (1977). Ce questionnaire mesure les sentiments de compétence, de satisfaction et d'efficacité parentales. Plus le score est élevé, plus le sentiment de compétence du parent est élevé. Le score est subdivisé en cinq zones allant d'un score très faible à un score très élevé. Cet outil est intéressant car il permet de mettre en évidence les deux dimensions de la compétence parentale, à savoir l'efficacité et la satisfaction. Il va donc être utile pour évaluer les sentiments de compétence et de satisfaction parentales du parent face à cet enfant victime. Le score du sentiment de compétence parentale consiste en l'addition de ces deux sous-échelles et varie de 16 à 96.

\subsubsection{L'Inventaire du Désir Sexuel (SDI)}

Cette échelle a été élaborée en français par Spector, Carey, et Sternberg (1996). Cet inventaire évalue le désir sexuel individuel et dyadique, en fréquence et en intensité. Cet outil a permis de tester l'hypothèse d'une baisse de l'intimité des parents à la suite de la découverte de l'abus sexuel, d'autant plus que cette composante du couple fait directement résonance avec l'expérience traumatique de l'enfant. Nous avons arbitrairement déterminé un score seuil qui nous a servi de point de repère. Pour ce faire, nous avons pris la médiane des scores, soit 45,5 ; seuil en-dessous duquel nous avons considéré le désir comme bas et au-dessus duquel comme élevé.

\subsection{Analyses}

\subsubsection{Analyse qualitative}

$\mathrm{Au}$ vu de la petite taille de l'échantillon et de l'objectif de cette étude, nous avons utilisé une analyse qualitative du type analyse de cas qui nous a permis de relater le récit du vécu des couples rencontrés en se focalisant principalement sur leur dynamique de couple. De plus, nous tirions des informations complémentaires grâce aux questionnaires administrés, non seulement une information chiffrée nous permettant de mesurer la dimension évaluée mais aussi une investigation plus fine et sensible des items qui ressortaient chez les participants. Nous avons porté notre intérêt sur la dynamique du couple et avons adopté un point de vue plus nuancé. Ainsi, nous avons proposé une liste de facteurs pouvant influencer positivement ou négativement le vécu des couples. D'abord en proposant une liste par couple à partir des analyses de cas et ensuite en regroupant les points communs entre les couples, dans un souci de tirer le commun du singulier. Ces facteurs peuvent avoir été explicités clairement par les couples ou sont tirés de nos hypothèses à la suite des analyses de cas. Ils sont également parfois vécus par un seul couple, parfois par plusieurs et parfois par tous. Les analyses de cas ne seront pas présentées dans leur entièreté pour des raisons de confidentialités. 


\subsubsection{Analyse quantitative}

Malgré la taille de notre échantillon, nous avons réalisé des analyses statistiques à l'aide du logiciel STATISTICA 12. Nous avons opté pour un test non-paramétrique pour échantillons appariés (test de Wilcoxon) en prenant les différentes mesures chez la mère comme étant appariées aux même mesures chez le père que nous considérions comme la mesure répétée.

\section{Résultats}

Nous exposerons brièvement les résultats obtenus aux questionnaires par les couples. Nous pouvons remarquer que chez chaque couple, le Questionnaire d'Auto-Évaluation de la Compétence Éducative et Parentale évaluant le sentiment de compétence ainsi que la satisfaction parentales sont d'un bon, voire d'un très bon niveau. De plus, l'Inventaire d'Alliance Parentale montre également des scores élevés. Les parents ont l'impression de former une bonne équipe dans l'éducation de leur(s) enfant(s). Au niveau des dimensions faisant partie du sous-système conjugal, les résultats sont moins unanimes. En effet, nous pouvons remarquer qu'il existe des disparités entre les couples mais aussi au sein même des couples. Nous pouvons faire l'hypothèse que des ressentis différents à ces dimensions (satisfaction conjugale, coping dyadique et désir sexuel) peuvent créer des tensions au sein du couple conjugal. Les analyses statistiques viennent appuyer cette hypothèse en montrant que la seule différence statistiquement significative est le niveau de désir sexuel entre mère et père $(Z=2,20 ; p=0,02)$ (Tableau 1$)$.

À un niveau davantage qualitatif, nous pouvons faire émerger de leur discours un ensemble de facteurs facilitant et aggravant le vécu des parents d'enfants ayant été abusés sexuellement. Ces facteurs modulent donc positivement ou négativement la détresse vécue par les parents. Premièrement, nous pouvons citer le soutien d'une équipe professionnelle. De manière unanime, les parents s'accordent à dire que les équipes SOS-Enfants constituent réellement une aide précieuse. En effet, elles rassurent sur l'état de santé mentale de l'enfant à la suite de l'abus sexuel vécu, sont empathiques envers les parents et ne les mettent pas en cause. En particulier, le fait que les équipes SOS-Enfants ne mettent aucune séquelle en avant pour l'enfant victime, aide les parents à relativiser. Ils peuvent dire que c'est le seul endroit où ils se sentaient écoutés par rapport à leurs inquiétudes liées à l'enfant. Cependant, pour un couple (couple 5), l'équipe n'a pas permis d'éclairer la situation de l'abus. Laissant le doute, les parents se disent satisfaits de la prise en charge mais inquiétés par le manque de réponses. Le fait que ces six couples fassent appel à une équipe SOS-Enfants pourrait témoigner d'une sensibilité importante au vécu de l'enfant mais également d'un souhait de se décharger des préoccupations engendrées par la problématique ce qui a donc pu créer un biais dans la sélection des participants et leur représentativité.

Deuxièmement, pour ceux qui ont entamé des procédures judiciaires (couples 2, 3 et 6), pour deux d'entre eux, cellesci se révèlent décevantes et très difficiles à vivre. Le troisième couple étant au tout début des procédures. En effet, ils ne se sentent pas entendus et les compensations proposées sont, pour eux, inadaptées. De fait, les parents sont en demande de protection et de reconnaissance pour leur enfant (punition de l'auteur, mise à l'écart,...) mais en aucun cas, la compensation financière n'est souhaitée ni jugée adéquate. Les parents se sentent remis en cause par la justice et la partie adverse. De plus, ils sont anxieux lorsque la date des audiences approche. Pour les couples ayant entrepris des procédures judiciaires et étant toujours en cours au moment des rencontres (couples 2 et 3), les pères semblent les plus affectés et en difficulté par rapport à celles-ci. Ils sont en colère et déçus de ces procédures. Cependant, pour les parents, ces procédures étaient primordiales. Ils voient en ces démarches la façon de rendre honneur à leur enfant et de le protéger. L'engagement dans de telles procédures chercherait à combler, en quelque sorte, l'échec de protection qui fait suite à la révélation. Notons que dans notre échantillon, parmi les trois couples n'ayant pas entamé de procédures judiciaires, deux concernent des abus intrafamiliaux dans la fratrie. Les parents estiment alors inenvisageable de porter plainte contre l'un de leurs enfants. Le dernier couple n'ayant pas porté les faits en justice l'explique par la minorité de l'abuseur et par l'empathie éprouvée envers lui. De plus, ils ne souhaitaient pas être contraints d'en reparler à divers intervenants. Ainsi,

Tableau 1

Résultats aux questionnaires.

\begin{tabular}{|c|c|c|c|c|c|}
\hline Couples & DAS & DCI & QAECEP & PAI & SDI \\
\hline \multirow[t]{2}{*}{1} & Mère : 87 & Mère : 92 & Mère : 63 & Mère : 80 & Mère : 41 \\
\hline & Père : 114 & Père : 97 & Père : 73 & Père : 92 & Père : 44 \\
\hline \multirow[t]{2}{*}{2} & Mère : 105 & Mère : 99 & Mère : 83 & Mère : 76 & Mère : 53 \\
\hline & Père : 108 & Père : 116 & Père : 78 & Père : 88 & Père : 71 \\
\hline \multirow[t]{2}{*}{3} & Mère : 102 & Mère : 108 & Mère : 64 & Mère : 90 & Mère : 21 \\
\hline & Père : 101 & Père : 111 & Père : 62 & Père : 85 & Père : 37 \\
\hline \multirow[t]{2}{*}{4} & Mère : 98 & Mère : 103 & Mère : 73 & Mère : 83 & Mère : 28 \\
\hline & Père : 94 & Père : 104 & Père : 74 & Père : 83 & Père : 70 \\
\hline \multirow[t]{2}{*}{5} & Mère : 91 & Mère : 129 & Mère : 67 & Mère : 77 & Mère : 30 \\
\hline & Père : 76 & Père : 107 & Père : 69 & Père : 81 & Père : 49 \\
\hline \multirow[t]{2}{*}{6} & Mère : 116 & Mère : 123 & Mère : 74 & Mère : 94 & Mère : 43 \\
\hline & Père : 113 & Père : 131 & Père : 69 & Père : 88 & Père : 55 \\
\hline \multirow[t]{2}{*}{ Test de Wilcoxon pour échantillons appariés } & $Z=0,41$ & $Z=0,94$ & $Z=0,67$ & $Z=0,20$ & $Z=\mathbf{2 , 2 0}$ \\
\hline & $p=0,67$ & $p=0,34$ & $p=0,50$ & $p=0,83$ & $p=\mathbf{0 , 0 2}$ \\
\hline
\end{tabular}

Les résultats en gras sont ceux qui se trouvent en-dessous du seuil critique. 
ce couple montrait moins d'inquiétudes, entre autre, selon eux, parce que l'abuseur était mineur. Pour certains (couples 5 et 6), le médecin traitant aide car il est source de support social et peut apporter une aide médicamenteuse, principalement aux mères, comme des somnifères.

Ensuite, les caractéristiques de l'abus semblent venir moduler le vécu des couples. Le caractère intrafamilial serait moins facile à gérer. Certains couples disent d'ailleurs que si l'auteur des faits avait été une personne extérieure, la situation aurait été plus facile à vivre (couples 3, 4, 5 et 6). En effet, cette dimension engendre des pertes relationnelles sans doute plus importantes mais aussi le développement de réactions parfois plus ambiguës envers l'auteur et la victime. Cet aspect apparaît de manière encore plus complexe lorsqu'il s'agit de fratries (couples 4 et 5), d'autant plus si un éloignement du milieu familial a eu lieu (couple 5). Dans ce cas, les mères se sentent mises en cause, remettent en question leur éducation, et ce, plus que les pères. La nature de l'abus entre également en compte : les abus graves (répétés et pénétrations) affecteraient plus les parents (couples 2 et 6). Lorsqu'ils n'ont aucune information concernant la durée des faits, ils tenteraient de se rassurer en la limitant dans le temps (couples 4 et 5). Enfin, les parents se montrent plus en colère lorsque l'auteur des faits les nie (couples 2, 3 et 6).

L'évitement et le déni semblent être largement utilisés par tous les couples. À court terme, ces mécanismes défensifs permettraient aux parents de continuer à vivre sans trop de difficultés. Cependant, à plus long terme, ceux-ci ne s'avèrent pas adaptatifs car ils empêchent de dépasser le trauma. Pour ces personnes ayant tendance à « oublier », à « ne prendre que le bon côté », à « ne plus en parler »,... nous pouvons imaginer que la survenue d'évènements les confrontant à nouveau au trauma (médias, même prénom, ressemblance, situation similaire,...) réactiverait celui-ci, en raison de la non-élaboration. En effet, l'évitement et le déni sont des mécanismes de défense qui contribuent à mettre de côté, sans élaborer l'évènement traumatique.

Le support social peut être trouvé auprès de diverses personnes : la famille, les amis et les collègues. Dans tous les cas, lorsqu'il y a support social, les parents se sentent soutenus et aidés. Entretenir des relations sociales avec des personnes qui ne sont pas au courant des faits peut également être bénéfique (couples 1, 3, 4 et 6). Cela permettrait de ne pas être jugé et de pouvoir reprendre, en partie tout du moins, le cours de la vie comme si de rien n'était. Par contre, être remis en cause, vivre des ruptures de liens ou s'isoler socialement ne semblent pas aider ces couples (couples 2, 3, 4, 5 et 6).

En ce qui concerne la dynamique familiale, lorsque la fratrie est constituée de plusieurs enfants et non uniquement de l'enfant victime (couples 2, 3 et 5), les parents tendent à considérer l'enfant n'étant pas victime comme une ressource. Ce dernier leur permettrait de continuer à avancer. Cependant, ce phénomène peut constituer un risque pour l'enfant «salvateur ». En effet, être celui qui soutient ses parents peut être un rôle difficile à jouer. Si ce mécanisme persiste, nous y voyons un risque de parentification. Les couples ayant un enfant unique (couple 1) ou ayant deux fils victimes (couple 6) se focalisent plus sur le problème de l'enfant et ont tendance à le surprotéger.
Concernant la dynamique du couple, les couples qui se montrent en cohésion (couples 1, 3 et 6), c'est-à-dire qui sont en accord sur les démarches à entreprendre, dont un des membres s'investit plus à la suite de cette révélation semblent trouver un équilibre contrairement à ceux qui manquent de cohésion ou qui présentent des disparités importantes dans les diverses dimensions évaluées (couples 2,4 et 5). En ce qui concerne la satisfaction conjugale, certains couples parlent d'une augmentation de la communication (couples 3 et 6 ) et nous pouvons observer qu'un niveau satisfaisant dans la relation conjugale maintient et aide les couples (couples 2, 3 et 6) contrairement à une mise entre parenthèses de la vie de couple et/ou d'un rapprochement du couple autour de l'enfant (couples 1, 2, 3 et 6). Au niveau du coping dyadique, avoir la même approche de la gestion du stress mais aussi respecter les besoins et espaces de chacun aide les couples à se soutenir (couples 1, 3 et 6), contrairement à une approche divergente qui tend à les éloigner et les pousse à aller chercher de l' aide ailleurs (couples 2, 3, 4 et 5). Au niveau parental, l'alliance et le sentiment de compétence parentale sont d'un bon, voire d'un très bon niveau pour les couples rencontrés et cette dimension pourrait être une ressource. Cependant, à la suite de la révélation, tous les couples culpabilisent et se remettent en question. Ainsi, dans un premier temps, le vécu des parents est aggravé par ces sentiments. Enfin, en ce qui concerne le niveau de désir sexuel, pour certains couples (couples 3 et 6), l'intimité apporte réconfort et mène au rétablissement d'un certain équilibre conjugal. Cependant, le dégoût et les disparités dans le niveau de désir sont majoritaires et peuvent être source de tensions dans la relation conjugale (couples 1, 2, 4 et 5 ). Ainsi, le tableau de résultats montre que 5 couples sur 6 manifestent soit un faible niveau de désir sexuel, présent chez chaque membre du couple (couples 1 et 3 ), soit une disparité importante entre les deux partenaires (couples 4, 5 et 6), la mère ayant à chaque fois un niveau de désir sexuel moins élevé que le père. Un seul couple (couple 2) met en évidence un niveau de désir sexuel au-dessus du score établi. De cette façon, nous observons que la sexualité de ces couples, dimension intégrant la conjugalité, est mise en péril.

\section{Discussion}

Si nous reprenons notre hypothèse générale qui consistait à supposer une détresse significative au sein du couple après l'annonce de l'abus sexuel vécu par un de leur enfant, il va sans dire que celle-ci se confirme nettement. Ces résultats rejoignent ceux d'autres études quantitatives reposant sur une population importante $[3,10,11]$. Cependant, notre étude révèle que cette détresse s'exprime de façon différente non seulement entre tous les couples rencontrés mais également au sein des couples euxmêmes. De plus, elle est modulée par un certain nombre de facteurs que nous venons d'exposer dans les résultats. Au sein du couple conjugal, nos hypothèses, à savoir une insatisfaction conjugale, des difficultés d'ajustement dyadique et une baisse de l'intimité, ne se vérifient que partiellement car nous n'observons jamais le profil supposé chez chaque couple et certains facteurs externes à la problématique de l'abus peuvent expliquer les résultats observés (instabilité du couple, dépression 


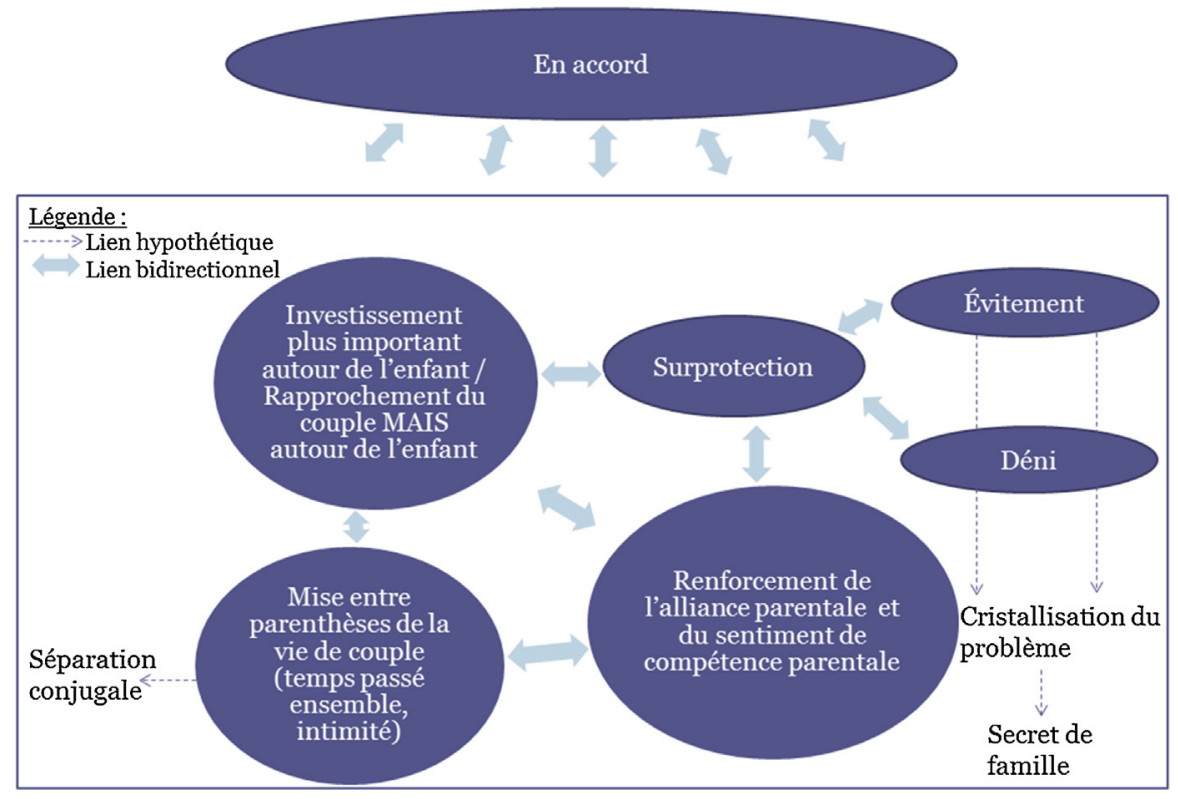

Fig. 1. Modélisation du processus conjugal et parental vécu par les parents d'enfants abusés sexuellement à la suite de la révélation.

saisonnière, surcharge de travail,...). Par contre, au niveau du couple parental, les hypothèses, à savoir une insatisfaction parentale, un sentiment d'incompétence parentale et enfin un affaiblissement de l'alliance parentale, sont toutes infirmées. Ainsi, le sous-système parental agirait davantage comme une ressource et contribuerait à diminuer, dans un premier temps, le vécu de détresse de ces couples. En effet, par l'investissement autour de l'enfant, les parents sont préoccupés par son bien-être au détriment d'une attention particulière à leur vécu de détresse conjugale. Par ailleurs, sur base de l'analyse qualitative de nos résultats, nous proposons une modélisation du processus pouvant être traversé par les couples dont un enfant a vécu un abus sexuel (Fig. 1).

Nous pouvons observer une mise entre parenthèses de la vie de couple (comme dans la plupart des évènements touchant les enfants : naissance, maladie,...). Celle-ci se concrétise par une diminution du temps passé ensemble, des sorties, de l'intimité,.. . Si nous poussons à l'extrême cet effacement conjugal, nous pouvons faire l'hypothèse que ce dernier pourrait conduire à une séparation conjugale comme deux de nos couples l'ont vécue (couples 2 et 5). Cependant, cette mise entre parenthèses a comme aspect positif un investissement plus important autour de l'enfant. Ce rapprochement semble avoir comme effet bénéfique, le renforcement de l'alliance parentale et du sentiment de compétence parentale qui sont d'ailleurs chez chaque couple d'un bon voire d'un très bon niveau. Sentiments qui ont été mis en cause à la suite de la révélation. Cependant, cet investissement autour de l'enfant engendre un processus de surprotection qui lui-même renforce la parentalité. Néanmoins, couplée à l'évitement et au déni, mécanismes souvent mis en place par les parents à la suite de la révélation de l'abus vécu par leur enfant, cela pourrait conduire à une cristallisation du problème, étant donné que l'élaboration du trauma est empêchée par ces mécanismes et à long terme, à la création d'un secret de famille lourd à porter. Enfin, les couples sont souvent en accord sur les démarches à entreprendre dans la gestion des révélations, ce qui renforce ce processus.

Nous illustrons ce modèle par une mise en concordance avec le vécu de chaque couple. Pour le couple 1, nous notons une incapacité à se décentrer de l'enfant. Cependant, il est enfant unique ce qui pourrait expliquer, en partie, cette attention portée sur lui. Dès que nous abordons une dimension essentiellement conjugale, Madame fait un lien avec leur enfant. Selon elle, son enfant n'a pas été traumatisé, donc eux non plus. Lorsque nous abordons la thématique de la sexualité, Madame fait référence à l'absence de pudeur de l'enfant. L'évènement vécu semble avoir été une problématique essentiellement vécue par l'enfant et prise en charge par un professionnel, ce qui met en évidence une extériorisation du problème par les parents. Cette caractéristique pourrait éclairer les résultats aux questionnaires. En effet, leur parentalité semble bien fonctionner alors que leur conjugalité est mise de côté au profit de l'enfant. Cependant, à la suite de la révélation, l'enfant s'est vu être surprotégé, ne pouvant plus aller dormir chez des amis, étant surveillé en permanence lors de ses activités,... Monsieur avait lui plutôt tendance à « vite oublier », « ne plus en parler » de façon à créer un climat d'évitement. Pour le couple 2, l'impact de la révélation de l'enfant semble avoir provoqué de grandes difficultés d'ordre conjugal mais aussi parental. En effet, ce couple a vécu une séparation, Madame n'était plus disponible pour investir la relation conjugale, se concentrant principalement sur les démarches à mettre en place pour protéger son enfant et Monsieur la laissant faire. Cette façon de gérer la situation a eu tendance à cristalliser le problème, madame, en se coupant de son ressenti et monsieur, en restant à l'écart, ils éprouvent toujours des difficultés à parler de l'évènement et semblent à nouveau envahis par l'évènement lorsqu'ils sont confrontés à ce sujet. Le couple 3 quant à lui est resté fort centré sur la gestion du problème au niveau parental sans réellement prendre en compte leurs émotions personnelles et leurs répercussions sur le niveau conjugal. Avant tout, ils 
désiraient protéger leur fille mais cette protection à outrance finit par enfermer leur enfant dans un secret important. Nous en savons très peu sur leur vie de couple, ce qui nous amène à penser que celle-ci n'est plus au centre de leur préoccupation. En effet, ils acceptent de participer à une recherche s'intéressant au vécu du couple tout en évitant d'en parler. Au niveau du couple 4, ils ont vécu chacun des réactions très différentes du point de vue de leur nature et de leur intensité. Bien que Monsieur dit avoir été là pour Madame, celle-ci ne s'est pas prononcée à ce sujet. Plusieurs éléments nous amènent à nous demander si elle a eu l'occasion d'exprimer sa souffrance. En effet, son époux avait tendance à banaliser fortement la situation, de façon à en dénier toutes conséquences. Cependant, à demi-mot, Madame pouvait dire que dans sa vie conjugale, elle se sentait moins satisfaite. De plus, une attention particulière était portée aux enfants afin que les faits entre eux ne se reproduisent plus, ce qui nécessite une surveillance de tous les instants laissant peu de place au répit en famille. Pour le couple 5, il semble que cette situation affecte très directement Madame qui ne peut s'investir dans sa relation de couple qui paraît tenir à un fil. Elle porte beaucoup de poids sur ses épaules et ne veut pas accepter d'aide, de sorte qu'elle s'attribue la faute des faits. Monsieur reste très extérieur face à la situation mais aussi dans leur relation conjugale qui semble compliquée par le vécu de Madame. Ils parlent peu d'eux-mêmes et une séparation conjugale a déjà eu lieu. Madame prend appui sur un de ses enfants, le plus jeune, mais les faits sont tabous dans la maison, de sorte à créer un climat de déni et donc de secret. Enfin, pour le couple 6, nous pouvons conclure que les partenaires sont plus soudés tout en laissant de côté leur vie conjugale. Ils se sont rapprochés pour leurs fils afin de les soutenir et les protéger. Cependant, ce changement semble les satisfaire et ils paraissent être actuellement dans un besoin de renfermement des frontières sur leur famille nucléaire.

Maintenant que nous avons exposé ce modèle et que nous l'avons illustré brièvement par les situations, il convient de chercher une explication à chaque phénomène observé. Abordons premièrement, la séparation conjugale. Minuchin nous rappelle l'importance pour le couple conjugal de conserver un « territoire psychosocial », c'est-à-dire un réseau social mais aussi un soutien affectif à deux ([22], p.73). Dans ce cadre, si le couple s'efface, nous pouvons comprendre que sans cet espace de soutien affectif du couple, la séparation conjugale devient envisageable. Dans nos analyses de cas, nous n'observons pas une insatisfaction conjugale de façon systématique chez chaque couple. En effet, dans leurs discours, les couples se montrent globalement satisfaits. Il semblerait que le moment de crise ne soit que temporaire et ne se répercute dès lors pas à long terme sur la qualité de la relation conjugale. Cependant, pour trois des couples rencontrés, un membre (couples 1 et 4 ) ou les deux (couple 5) présentent une insatisfaction de leur relation conjugale comme le montrent les réponses aux questionnaires. Ce modèle met également en lumière une tendance des couples à la focalisation autour de l'enfant. Ce mécanisme apparaît comme rassurant et adaptatif et semble répondre à un désir de réparation qui fait suite au sentiment d'avoir failli à son rôle protecteur. Ce résultat est également partagé par Scheeringa et Zeanah [18]. Au niveau du coping dyadique, nos observations montrent que les parents sont très souvent en accord sur les démarches à entreprendre autour de l'enfant, ce qui nous apparaît comme une ressource. Cependant, la gestion des émotions (repli-communication) est bien souvent différente et tend à éloigner les membres du couple et les pousse à aller chercher du soutien ailleurs (famille élargie, médecin traitant, amis,...). La focalisation sur l'enfant a en outre comme conséquence une diminution de l'intimité du couple, qui peut s'accentuer par le fait que la vie sexuelle fait directement écho à l'expérience traumatique vécue par l'enfant. La sexualité peut provoquer du dégoût chez un ou les deux parents et contribue ainsi à un éloignement des partenaires du couple.

Bien que l'objectif principal ait été rencontré, à savoir explorer les difficultés des couples dont un enfant ou plusieurs ont vécu un abus sexuel, nos hypothèses n'ont été que partiellement vérifiées. Nos résultats hétérogènes s'expliquent certainement par la diversité des circonstances de l'abus mais aussi des systèmes et fonctionnements familiaux. Ils ont ainsi le mérite de refléter la réalité de l'abus et de ses conséquences dans sa finesse et sa complexité, trop souvent gommées par les analyses statistiques globales sur de plus larges échantillons. Chaque famille étant unique et ayant ses propres ressources, la détresse s'exprime de façon différente, tant dans son intensité que dans sa temporalité. Si nous ne connaissions que très peu l'état antérieur de la relation conjugale, il semblerait toutefois que les couples rencontrés ne présentaient pas de difficultés conjugales majeures avant la révélation. Cette hypothèse est soutenue par les résultats de Manion et al. [2] qui avancent que, dans une relation conjugale en difficulté, le dévoilement agit comme un facteur aggravant. Les couples rencontrés semblent, dans la majorité, disposer de nombreuses ressources et mettent en évidence une capacité adaptative à faire face à la situation. Au-delà, certains couples expriment un renforcement des liens. Ce constat est également partagé par Karney et ses collègues, 2005 [21] qui proposent l'idée que dans une relation satisfaisante, la capacité à faire face à un évènement potentiellement traumatique peut renforcer les liens des partenaires du couple par le soutien affectif qu'ils s'apportent mutuellement. Cependant, deux couples rencontrés apparaissent plus en difficulté dans leur relation conjugale (couples 4 et 5). Dans une relation déjà fragile, un évènement de vie majeur peut conduire à la détresse conjugale [21].

Il nous semble important de discuter des limites de cette recherche afin de nuancer les résultats obtenus. Premièrement, nos résultats ont été récoltés de façon rétrospective. Ainsi, ils sont reconstruits et certainement teintés d'un grand nombre de défenses. De plus, comme nous l'avons évoqué, la désirabilité sociale a certainement joué un rôle, réduisant l'authenticité du discours, qui laisse apparaître une relation conjugale d'assez bonne qualité et cohésive, tandis que les questionnaires mettent en évidence davantage de difficultés et une différenciation des membres du couple. Une deuxième limite à énoncer est inhérente au cadre proposé. En effet, les entretiens sont limités dans le temps, ce qui ne nous laisse pas l'occasion d'installer un rapport de confiance. Par ailleurs, la présence simultanée des deux membres du couple a pu freiner chacun des partenaires à partager son vécu réel. Ensuite, en raison tout d'abord de la petite taille 
de notre échantillon, nous ne pouvons prétendre à une généralisation de nos résultats. De plus, nous n'avons pas tenu compte du niveau de gravité de l'abus dans la modélisation du processus. Nous ne pouvons en effet pas mettre sur le même pied les diverses formes d'abus existantes, qui peuvent être d'intensités variables. Enfin, ces couples font tous appel à une équipe SOSEnfants, ce qui témoigne d'une certaine sensibilité au vécu de leur enfant mais peut-être aussi d'une anxiété importante par rapport à la problématique sexuelle.

Malgré les limites citées précédemment, cette méthodologie trouve son intérêt car elle va à la recherche de l'hétérogénéité et de la compréhension de la complexité de réalités diverses. Elle va directement à la rencontre des vécus et permet aux couples de s'exprimer et d'être écoutés. De plus, nous utilisons des échelles traitées de façon qualitative et quantitative. La taille de l'échantillon ne permet de procéder qu'à des analyses statistiques limitées mais les valeurs sont utilisées pour situer ces couples par rapport au score seuil. Nous ne nous limitons cependant pas à la valeur chiffrée, nous avons également utilisé les items dans leur version qualitative. Les couples étaient satisfaits d'avoir participé. Cette démarche les amène aussi à découvrir leur vécu respectif. Celui-ci étant souvent peu exprimé, ils peuvent se rendre compte de ce que l'autre vit. En effet, peu de place est faite à la souffrance parentale et conjugale dans les dispositifs en place actuellement autour de l'abus sexuel chez l'enfant. Myrick et Green [17] mettent en évidence que les parents d'enfants victimes sont aussi traumatisés, bien qu'ils minimisent les faits pour avant tout protéger les enfants. Il apparaît comme évident pour eux de faire passer la protection et le bien-être de l'enfant avant tout, en laissant ainsi, le couple de côté.

D'un point de vue clinique, cette étude souligne l'importance qu'il y a à soutenir et à restaurer la parentalité afin que les parents, dans une telle situation de crise, puissent percevoir et activer cette dimension comme une ressource. Nous savons en effet combien un co-parentage négatif peut être lié à l'apparition de difficultés comportementales chez l'enfant [23]. D'autre part, cette étude montre l'importance de soutenir aussi la conjugalité et l'intimité du couple, trop souvent oubliées. Bien que nous observions souvent un effacement du couple suite aux révélations d'abus par l'enfant, celui-ci est adaptatif et primordial, pour autant qu'il s'établisse sur le court terme. Ce processus nécessite en effet un réajustement et une mise en mouvement une fois la période aiguë dépassée. Ces couples ne sont généralement pas préparés à vivre une telle prise de distance et n'ont pas toujours conscience du caractère fonctionnel de celle-ci. Le résultat significatif concernant la sphère sexuelle dans nos données montre le plus gros point de discordance au sein des couples qui peut être à long terme une source de problème. Un travail de sensibilisation et de recherche de sens pourrait contribuer à normaliser leur vécu. Delage [24] nous rapporte que les familles dont un membre a vécu un traumatisme ont elles-mêmes besoin d'être contenues. En effet, par le principe de circularité, la souffrance de la victime retentit sur le fonctionnement des couples mais aussi des familles. Le traumatisme fait effraction dans la vie familiale et altère, en quelque sorte, le système de pareexcitation. Ainsi, par des interventions contenantes, le système peut se reconstruire. Dans cette perspective, il serait intéressant de créer un espace de parole pour chaque sous-système, pour les parents et pour l'enfant victime mais aussi les fratries. On sait par ailleurs combien les enfants peuvent être réticents à dévoiler pour ne pas inquiéter leurs parents [15]. De cette façon, une prise en charge et une attention à chaque sous-système devraient permettre de diminuer la détresse de chacun. Cependant, les couples ne semblent pas disponibles pour eux dans un premier temps. Il nous paraît important que les thérapeutes soient particulièrement attentifs et sensibles au vécu de ces couples, sachant, de surcroît, que ceux-ci tendent à s'effacer et à se mettre de côté, laissant toute la place à l'enfant et à sa protection. Ainsi, dans le respect de la temporalité de la crise familiale, il serait intéressant de mettre en place une prise en charge en deux temps : l'attention serait d'abord portée à l'enfant victime et dans un deuxième temps seulement, en parallèle à la prise en charge de l'enfant, un espace serait ouvert et accordé au couple. Cependant, les disparités entre le discours et les résultats issus des questionnaires posent la question de la pertinence des entretiens de couples et de leur liberté à se différencier du récit commun, dans une loyauté aux sous-systèmes conjugal et parental. Des espaces individuels pour chacun des parents pourraient donc être proposés.

\section{Conclusion et perspectives}

Cette étude montre l'intérêt de mettre en place une série d'évaluations permettant de mesurer la détresse conjugale et individuelle des parents mais aussi de soutenir et valoriser les ressources parentales, et ce, à divers moments clés : au moment de la révélation, après la prise en charge de l'enfant, pendant les éventuelles procédures judiciaires et quelques années après. Ainsi, une recherche longitudinale pourrait approcher sur du plus long terme le type de fonctionnement de ces familles, et les réponses mises en place aux différents temps de cette révélation.

Pour conclure, nous retenons l'impact important que la révélation d'un abus sexuel vécu par un enfant peut avoir sur le couple non seulement parental mais aussi conjugal. Bien que nous mettions en avant le couple parental comme une ressource, le couple conjugal est mis en péril. Or l'enfant est sensible au vécu de ses parents et peut ressentir les tensions au sein du couple conjugal, ce qui peut augmenter les sentiments de culpabilité et/ou entraver les processus de résilience face à l'abus sexuel. De plus, à long terme que devient cette dimension du couple ? Il nous apparaît alors important de continuer à s'intéresser à mieux comprendre le vécu de ces couples mais aussi l'influence de celui-ci sur la détresse des enfants victimes.

\section{Déclaration de liens d'intérêts}

Les auteurs déclarent ne pas avoir de liens d'intérêts.

\section{Références}

[1] Grosz CA, Kempe RS, Kelly M. Extrafamilial sexual abuse: treatment for child victims and their families. Child Abuse Neglect 2000;24:9-23, http://dx.doi.org/10.1016/S0145-2134(99)00113-1. 
[2] Manion G, McIntyre J, Firestone P, Ligezinska M, Ensom R, Wells G. Secondary traumatization in parents following the disclosure of extrafamilial child sexual abuse: initial effects. Child Abuse Neglect 1996;20:1095-109, http://dx.doi.org/10.1016/0145-2134(96)00098-1.

[3] Kaléidos asbl. Recommandations dans le travail avec les parents non abuseurs d'enfants victimes d'abus sexuel intra-familial : comment aider les parents d'un enfant abusé sexuellement dans sa famille ? Liège, Belgique; 2008 [Retrieved from http://www.asblkaleidos.be/page3.html].

[4] Barudy J. La douleur invisible de l'enfance : approche éco-systémique de la maltraitance (chap. 6, 7, 8 \& 9). Toulouse, France: Eres; 2007.

[5] Tourigny M, Baril K. Les agressions sexuelles durant l'enfance : ampleur et facteurs de risque. In: Hébert M, Cyr M, Tourigny M, editors. Les agressions sexuelles envers les enfants (Tome 1). Québec, Canada: Presses de l’Université du Québec; 2011. p. 7-50.

[6] Tourigny M, Hébert M, Joly J, Cyr M, Baril K. Prevalence and cooccurrence of violence against children in the Quebec populaton. Aust N Z J Public Health 2008;32(4):331-5.

[7] Martin P, Van Poppel E. Dévoilement et signalement d'un abus sexuel. In: Born M, Delville J, Mercier M, Sand EA, editors. Les abus sexuels d'enfants : interventions et représentations. Bruxelles, Belgique: Mardaga; 1996. p. 35-61.

[8] Palmer SE, Brown RA, Rae-Grant NI, Loughlin MJ. Responding to children's disclosure of familial abuse: what survivors tell us. Child Welfare 1999;78(2):259-82 [Retrieved from https://vpn.gw.ulg.ac.be/,DanaInfo= search.proquest.com+?accountid=14630].

[9] Spaccarelli S. Stress, appraisal, and coping in child sexual abuse: a theoretical and empirical review. Psychol Bull 1994;116:340-62, http://dx.doi.org/10.1037/0033-2909.116.2.340.

[10] Davies MG. Parental distress and ability to cope following disclosure of extra-familial sexual abuse. Child Abuse Neglect 1995;19(4):399-408, http://dx.doi.org/10.1016/0145-2134(95)00010-6.

[11] Dyb G, Holen A, Steinberg AM, Rodriguez N, Pynoos RS. Alleged sexual abuse at a day care center: impact on parents. Child Abuse Neglect 2003;27:939-50, http://dx.doi.org/10.1016/S0145-2134(03)00141-8.

[12] Kilroy SJ, Egan J, Maliszewska A, Sarma KM. "Systemic trauma": the impact on parents whose children have experienced sexual abuse. J Child Sex Abus 2014;23(5):481-503, http://dx.doi.org/10.1080/ 10538712.2014.920458.
[13] Cyr M, Zuk S, Payer M. Le profil et l'évaluation des parents dont les enfants ont été agressés sexuellement. In: Hébert M, Cyr M, Tourigny M, editors. Les agressions sexuelles envers les enfants (Tome 1). Québec, Canada: Presses de l'Université du Québec; 2011. p. 253-302.

[14] Elliot AN, Carnes CN. Reactions of nonoffending parents to the sexuel abuse of their child: a review of the literature. Child Maltreat 2001;6:314-31, http://dx.doi.org/10.1177/1077559501006004005.

[15] Hébert M. Les profils et l'évaluation des enfants victimes d'agression sexuelle. In: Hébert M, Cyr M, Tourigny M, editors. Les agressions sexuelles envers les enfants (Tome 1). Québec, Canada: Presses de l’Université du Québec; 2011. p. 149-204.

[16] Van Toledo A, Seymour F. Interventions for caregivers of children who disclose sexual abuse: a review. Clin Psychol Rev 2013;33:772-81, http://dx.doi.org/10.1016/j.cpr.2013.05.006.

[17] Myrick AC, Green EJ. A play-based treatment paradigm for nonoffending caretakers: evidence-informed secondary trauma treatment. Int J Play Ther 2013;22(4):193-206, http://dx.doi.org/10.1037/a0034560.

[18] Scheeringa MS, Zeanah CH. A relational perspective on PTSD in early childhood. J Trauma Stress 2001;14(4):799-815 [0894-9867/01/10007W119.50].

[19] Terrisse B, Trudelle D. Le questionnaire d'auto-évaluation de la compétence éducative parentale. Université du Québec, Montréal: Groupe de recherche en adaptation scolaire et sociale, Département des Sciences de l'Education; 1988.

[20] Baillargeon J, Dubois G, Marineau R. Traduction française de l'échelle d'ajustement dyadique. Can J Beh Sci 1986;18(1):25-34, http://dx.doi.org/10.1037/h0079949.

[21] Favez N. L'examen clinique du couple : théories et instruments d'évaluation. Bruxelles, Belgique: Mardaga; 2013.

[22] Minuchin S. Familles en thérapie (chap. 3). Paris, France: Erès; 1979.

[23] Valentino K, Berkowitz S, Stover CS. Parenting behaviors and posttraumatic symptoms in relation to children's symptomatology following a traumatic event. J Trauma Stress 2010;23:403-7, http://dx.doi.org/ $10.1002 /$ jts. 20525 .

[24] Delage M. Traitement familial du traumatisme psychique. Ther Fam 2000;21(3):273-87. 